\title{
The ENBIS-15 Quality and Reliability Engineering International Special Issue
}

Grazia Vicario $^{1}$ and Xavier Tort-Martorell ${ }^{23}$

The 2016 special issue of Quality and Reliability Engineering International provides an overview of contributions by active members of the European Network for Business and Industrial Statistics (ENBIS) network. Most papers in this special issue were presented at the 15th Annual Conference of the ENBIS (ENBIS-15) which took place in the historic city of Prague (Czech Republic) from $7^{\text {th }}$ to $9^{\text {th }}$ September 2015, with preand post-conference workshops. Antonio Pievatolo (CNR-IMATI: MILAN, Italy) and Bart De Ketelaere(University of Leuven, Leuven, Belgium) chaired the Program Committee and Gejza Dohnal (Czech Technical University, Prague)presided over the Organizing Committee.

The abstracts of all oral and poster presentations, in most instances accompanied by full papers and/or presentation slides, are available on the ENBIS web site (www.enbis.org).

Once again the ENBIS special issue covers theoretical and methodological advances in business and industrial statistics and shows the variety of topics that characterize the ENBIS community.

Taking advantage of the coincidence in place and time with the XXI IMEKO World Congress the ENBIS 15 was connected to it; this special issue, features two papers related to measurement. Akkerhuis and de Mast (1) consider the problem of measurement error of nominal measurements when there is no 'gold standard' and provide a method to estimate systematic and random error components, and Berni and Nikiforova (2) suggest a novel approach for analysing interlaboratory comparison data based on an error measurement model in order to consider both the measured values and their uncertainty.

Postelnicu, Raviv and Ben-Gal (3) propose a method to minimize the expected number of clicks required to reach a website page. The method proposed provides very good results with a relatively low computational cost.

\footnotetext{
${ }^{1}$ Department of Mathematical Sciences, Politecnico di Torino, Corso Duca degli Abruzzi, 24, 10129, Torino, Italy

${ }^{2}$ Departament Estadística i Investigació Operativa, Universitat Politècnica de Catalunya. Barcelona TECH, Avda. Diagonal, 647, 08028, Barcelona, Spain

${ }^{3}$ Correspondence to: Xavier Tort-Martorell, Departament Estadística i Investigació Operativa, Universitat Politècnica de Catalunya, Barcelona TECH, Avda. Diagonal, 647, 08028 Barcelona, Spain. E-mail: xavier.tort@upc.edu
} 
Motivated by test data from video images coming from drones, Avery, Orndorff, Robinson and Freeman (4) investigate the regularization for continuously observed covariated that resembles step functions.

Damblin, Keller, Barbillon and Pasanisi (5) propose a Bayesian method to evaluate and finally select computer codes modelling complex physical systems. Another paper dealing with the modelization of Computer Experiments is Meta-models in Computer Experiments: Kriging vs Artificial Neural Networks by Vicario, Craparotta and Pistone (6). The authors compare the performances of two of the most popular metamodels, Kriging (using two different approaches, a parametric one and a non-parametric based on experimental variograms, in the estimation of the correlation structure) and Artificial Neural Network, in order to state which one guarantees higher accuracy at a satisfactory cost in Computational Fluid Dynamics experiments providing energy loss in Low Pressure Turbines, for the aim of reducing the specific fuel consumption.

The goal of Browne, Looss, Le Gratiet, Lonchampt and Remy (7) in Stochastic simulators based optimization by Gaussian process metamodels- Application to maintenance investments planning issues is the optimization of industrial asset management strategies. They build a metamodel of the stochastic simulator for assessing the indicator of interest (Net Present Value). In the paper the quantile function of the stochastic simulator is emulated. In a Gaussian process framework, an adaptive design method (QFEI) is defined by extending the well-known EGO algorithm, for the aim of obtaining an 'optimal' solution using a small number of simulator runs regarding the case study considered.

Fasso, Toccu and Magno (8), motivated by a multiple profile monitoring problem of a steam sterilizer, introduce general multifunctional EWMA control charts. Another paper dealing with the process monitoring is A Multivariate Control Chart for Autocorrelated Tool Wear Processes by Harris, Triantafyllopoulos, Stillman and McLeay (9). Here the authors propose an innovative on-line monitoring procedure for fully automate metal cutting processes. In fact, the drawback of having premature tool breakage and heavy tool wear giving rise to substantial costs and increasing the risk of non-conforming items is nowadays overcome using modern sensor technology for acoustic emissions, sounds, spindle power and vibration of the tool during a cut. The proposed methodology, mostly based on multivariate autoregressive state space model, is illustrated by a case study of titanium alloy milling (a machining process used in the manufacture of aircraft landing gears) and is demonstrated to outperform alternative residual control charts in this application.

Dror (10) resorts to a Quality Function Deployment (QFD) matrix to highlight controllable and noise factors that have to be considered when running a simulated experiment for a service system. (Identify important factors for service simulation experiments using QFD). Service design is a form of conceptual design involving the planning and organizing people, infrastructure communication of a service with the goal of improving the performances. A computer experiment may simulate different scenarios; because the complete set of possible scenarios is very vast, a criterion based 
on the Mean Square Error is suggested here for selecting the vital service factors to be considered in the computer experiment, taking inspiration from the Taguchi threestage design procedure.

The paper Compositional Data Methods in Customer Survey Analysis by Vives-Mestres, Martín-Fernández and Kenett (11) faces the problem of evaluating the customer satisfaction measured by questionnaires with statements scored on an anchored scale. The authors present common compositional tools ( $T_{C}^{2}$ control charts and principal components among others) with an application to the annual customer satisfaction survey of the $A B C$ Company. The novelty consists in highlighting the advantage of the compositional approach in dealing with non-response, which turns into a difficulty when dealing with zeros.

The paper Correlation analysis in contaminated data by singular spectrum analysis by Rodrigues and Mahmoudvand (12) concerns the use of new approaches in the analysis of the correlation when there are outliers in the set of the data, because the standard Pearson correlation might be misleading and result in erroneous results. These approaches are based on a non-parametric method designed for analyzing time series data, the Singular Spectrum Analysis. A set real data on aluminum industry is considered for comparisons with robust hybrid filtering methods.

The reproducibility is one of the cornerstones of the methodologies in Statistics. The technique of coating of materials to prevent wear can be improved by high velocity oxygen fuel spraying. Unfortunately, a drawback of this technique is the lack of reproducibility. Kuhnt, Rehage, Becker-Emden, Tillmann, Wolfgang and Hussong (13) assume the measures of temperature and velocity as target variables in generalized function-on-scalar regression in the paper Residual Analysis in Generalized Functionon-Scalar Regression for an HVOF Spraying Process. They propose methods to perform residual analysis in this framework aiming at the detection of individual residual functions which deviate from the majority of residuals. They prove that these methods help in detecting anomalies in the process and hence improve the estimators. To overcome the inconvenience of inspecting the ordinary residual plots at each observed point, they suggest the use of functional depths and prove their finding with simulation studies.

And in the last paper How can SMEs benefit from Big Data? Challenges and a path forward the authors (Coleman, Goeb, Manco, Pievatolo, Reis and Tort-Martorell (14) offer a big data maturity model for SMEs as a first step towards an SME roadmap to data analytics. Starting from the illustration of the state-of-the-art of IT with respect to usability for SMSs, the authors discuss how SMSs may overcome the barriers preventing them from adopting existing solutions. They review the history of Total Quality Management to highlight the core aspects of implanting a new paradigm. The management perspectives and the role of maturity models in enhancing and structuring the adoption of data analytics in an organization are considered, with recommendations to help SMES develop their big data capability facilitating them in continuing as the engines of European industrial and business success. 
The Editors gratefully thank all the experts and colleagues who were so kind to provide reviews for the submitted manuscripts. Special thanks go to Mrs. Emma Missen from the Quality and Reliability Engineering International editorial office for her patience, help and support she gave us during each step of the preparation of this issue.

See you in Sheffield in 2016 and Naples in September 2017.

\section{References}

1. Akkerhuis TS, de Mast J. Quantifying the random component of measurement error of nominal measurements without a gold standard. Quality and Reliability Engineering International 2016; 32.

2. Berni R, Nikiforova ND. Measurement error models for interlaboratory comparison measurement data. Quality and Reliability Engineering International 2016; 32.

3. Postelnicu Z, Raviv T amd Ben-Gal I. Improving Websites' quality of service by shortening their browsing expected path length. Quality and Reliability Engineering International 2016:32.

4. Avery M, Orndorff M, Robinson T, Freeman L. Regularization for continuously observed ordinal response variables with piecewise-constant functional covariates. Quality and Reliability Engineering International 2016; 32.

5. Damblin G, Keller M, Barbillon P, Pasanisi A, Parent É. Bayesian model selection for the validation of computer codes. Quality and Reliability Engineering International 2016; 32.

6. Vicario G, Craparotta G, Pistone G. Meta-models in computer experiments: kriging vs artificial neural networks. Quality and Reliability Engineering International 2016; 32.

7. Browne T, looss B, Le Gratiet L, Lonchampt J, Remy E. Stochastic simulators based optimization by Gaussian process metamodels - application to maintenance investments planning issues. Quality and Reliability Engineering International 2016; 32.

8. Fassò A, Toccu M, Magno M. Functional control charts and health monitoring of steam sterilizers. Quality and Reliability Engineering International 2016; 32.

9. Harris K, Triantafyllopoulos K, Stillman E, McLeay T. A multivariate control chart for autocorrelated tool wear processes. Quality and Reliability Engineering International 2016; 32.

10. Dror S. Identify important factors for service simulation experiments using QFD. Quality and Reliability Engineering International 2016; 32.

11. Vives-Mestres M, Martín-Fernández JA, Kenett RS. Compositional data methods in customer survey analysis. Quality and Reliability Engineering International 2016; 32. 
12. Rodrigues PC, Mahmoudvand R. Correlation analysis in contaminated data by singular spectrum analysis. Quality and Reliability Engineering International 2016; 32.

13. Kuhnt S, Rehage A, Becker-Emden C, Tillmann W, Hussong B. Residual analysis in generalized function-on-scalar regression for an HVOF spraying process. Quality and Reliability Engineering International 2016; 32.

14. Coleman S, Goeb R, Manco G, Pievatolo A, Reis M, Tort-Martorell X. How can SMEs benefit from big data? Challenges and a path forward. Quality and Reliability Engineering International 2016:32. 